\title{
Successful treatment of a patient with an EGFR- mutated pulmonary adenocarcinoma and a history of uremia with erlotinib after gefitinib-induced kidney dysfunction: a case report
}

\author{
L. Zhou* and Z. Chen
}

Respiratory ward 2, PCCM, Affiliated Hospital of Zunyi Medical University, Zunyi City, 563003, China

\section{Case Report}

\section{*Corresponding authors: \\ Dr. Linmei Zhou, \\ E-mail:}

LinmeiZhou@outlook.com

Revised: April 2020

Accepted: April 2020

Int. J. Radiat. Res., July 2021; 19(3): $749-753$

DOI: $10.29252 /$ ijrr.19.3.749

\section{ABSTRACT}

\begin{abstract}
A 48-year-old male patient with a history of uremia was diagnosed with pulmonary adenocarcinoma based on a computed tomography (CT) scan and biopsy. In addition, an epidermal growth factor receptor (EGFR) exon 19 deletion was identified by gene detection. Surgical treatment was not acceptable because of his poor physical condition, so he was treated with gefitinib. Kidney function deteriorated and the serum urea nitrogen and creatinine levels were increased. Two weeks after discontinuing treatment with gefitinib, kidney function gradually returned to normal and was treated with erlotinib. This is an unusual case involving a patient with EGFR-mutated pulmonary adenocarcinoma and a history of uremia who was successfully treated with erlotinib after gefitinib-induced kidney dysfunction.
\end{abstract}

Keywords: Epidermal growth factor receptor, pulmonary adenocarcinoma, Erlotinib, gefitinib, kidney function.

\section{INTRODUCTION}

With the increase in cigarette smokers and deterioration in air quality, the incidence of lung cancer in China has been increasing, especially in females and the younger population (1,2). Non-small cell lung cancer (NSCLC), a main subtype of lung cancer, accounts for approximately $85 \%$ of all cases of lung cancer, and is considered to be a major threat to public health (3).

Surprisingly, $10 \%-60 \%$ of patients with NSCLC, especially pulmonary adenocarcinoma, have an epidermal growth factor receptor (EGFR) mutation (4,5). Therefore, monoclonal antibodies that suppress phosphorylation/ activation and stimulate internalization of EGFR by inhibiting the extracellular domain of EGFR, and EGFR tyrosine kinase inhibitors-(TKIs) have been developed to inhibit development of NSCLC $(6,7)$. Among the EGFR-TKIs, oral selective EGFR-TKIs (erlotinib and gefitinib) are widely used as first-line treatment for NSCLC who do not respond to platinum-based chemotherapy (8). Gefitinib is administered at an oral dose of $250 \mathrm{mg} / \mathrm{d}$ and is highly bound to plasma proteins $(90 \%)$. The metabolites of gefitinib are mainly excreted via the feces, while renal elimination accounts for $<4 \%$ of the administered dose. Erlotinib is administered at an oral dose of $150 \mathrm{mg} / \mathrm{d}$, has high binding with plasma proteins (95\%) and is eliminated mainly in the feces $(90 \%)$, while only a small percentage $(9 \%)$ is excreted in the urine (9). Therefore, there are many cases that indicate gefitinib and erlotinib can be safely administered to NSCLC patients without affecting kidney function; however, there are also some cases in which EGFR-TKI-related nephrotoxicity has been reported. For example, Wan etal. (10) reported that gefitinib therapy induced uremia and azotemia, and kidney function recovered within 2 days after discontinuing gefitinib therapy. Similarly, the present study reports a case of gefitinib-induced kidney dysfunction and successful treatment 
with erlotinib in a patient with EGFR-mutated pulmonary adenocarcinoma and a history of uremia.

\section{Case report}

A 48-year-old male was diagnosed with uremia and hypertension and underwent a kidney transplantation 4 years ago at the Southwest Hospital of the Army Medical University. Post-operatively he received hemodialysis three times per week and regular assessment of kidney function in our hospital. Additionally, he also received treatment with metoprolol (25 mg twice daily; AstraZeneca, Macclesfield, UK), nifedipinecontrolled release tablets $(30 \mathrm{mg} / \mathrm{d}$; Bayer Pharma AG, Wuppertal, Germany), and terazosin hydrochloride tablets $(2 \mathrm{mg} / \mathrm{d}$; SECCO Pharmaceuticals, Beijing, China). During this period, there were no significant abnormalities in kidney function. He was admitted to our hospital due to a progressive cough, polypnea, and chest congestion for 3 months. The patient did not complain of any other symptoms, such as fevers, hemoptysis, night sweats, weight loss, and/or fatigue. This patient had been smoking for 20 years ( 1 pack/d) and denies a history of alcohol consumption or relevant family history. A computed tomography ([CT] GE Healthcare, Milwaukee, WI, USA) scan revealed a mass in the left hilus pulmonis and multiple nodules in the right lung (figure 1A). Cytologic staining of transbronchial biopsy specimens were positive for carcinoma cells (thyroid transcription factor-1 [TTF-1], new aspartic proteinase A [NapsinA], and cytokeratin 7 [CK-7]; Zhongshan Goldenbridge Biotechnology Co., Beijing, China; figure 2). An EGFR exon 19 deletion was identified by gene detection. Surgical treatment was not an option because of his poor physical condition. Thus, he received $250 \mathrm{mg}$ of gefitinib (AstraZeneca, Macclesfield, UK) orally once daily. The respiratory symptoms, including the cough, polypnea, and chest congestion, improved 4 weeks after treatment with gefitinib. The pulmonary nodules completely regressed and the pleural effusion had not increased (figure 1B). Kidney function, however, deteriorated and the serum urea nitrogen and creatinine levels were increased (figure 3A, 3B). Therefore, gefitinib was discontinued, and the serum urea nitrogen and creatinine levels gradually normalized (figure 3A, 3B). After 2 weeks, the patient was treated with erlotinib (OSI Pharmaceuticals, Inc., Melville, NY, USA) $150 \mathrm{mg}$ orally once daily and kidney function was closely monitored. During treatment with gefitinib, there were no adverse effects on kidney function. Four months after treatment with gefitinib, the lung lesion remained stable based on a follow-up CT scan (figure 1C).
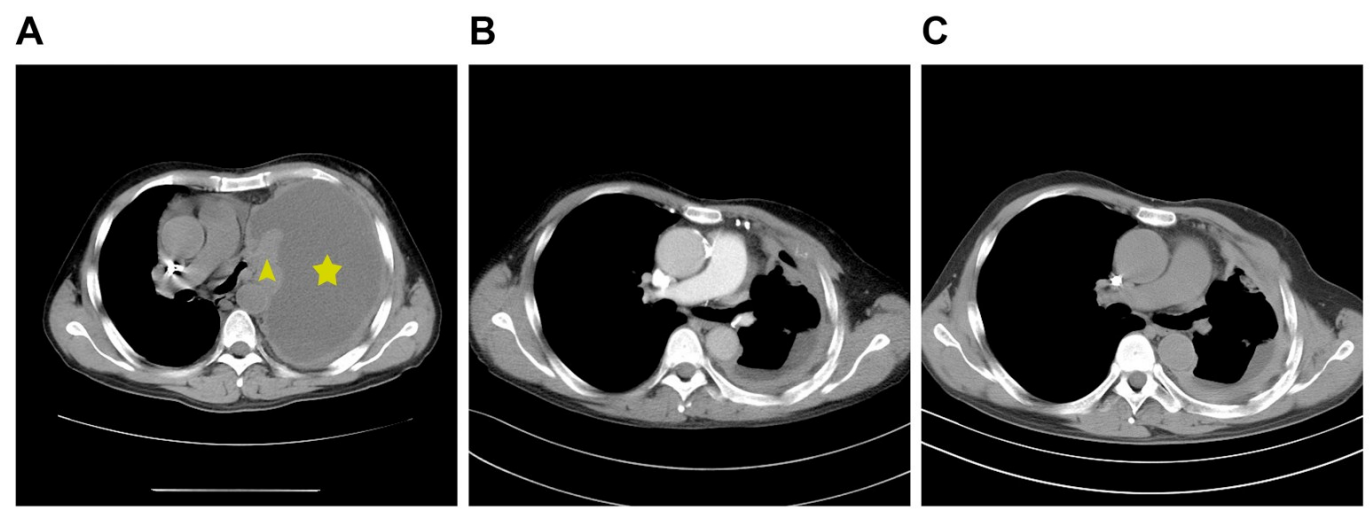

Figure 1. Follow-up chest computed tomography (CT) scan before and after gefitinib or erlotinib treatment. (A) Before the treatment, the CT scan shows there is a lump at the left hilus pulmonis (pointed by yellow triangle) and malignant pleural effusion in the left lung (pointed by yellow star). (B) After treatment with gefitinib for 4 weeks, or (C) subsequent treatment with erlotinib for 4 weeks, the lump completely regressed and the pleural effusion was significantly decreased. 

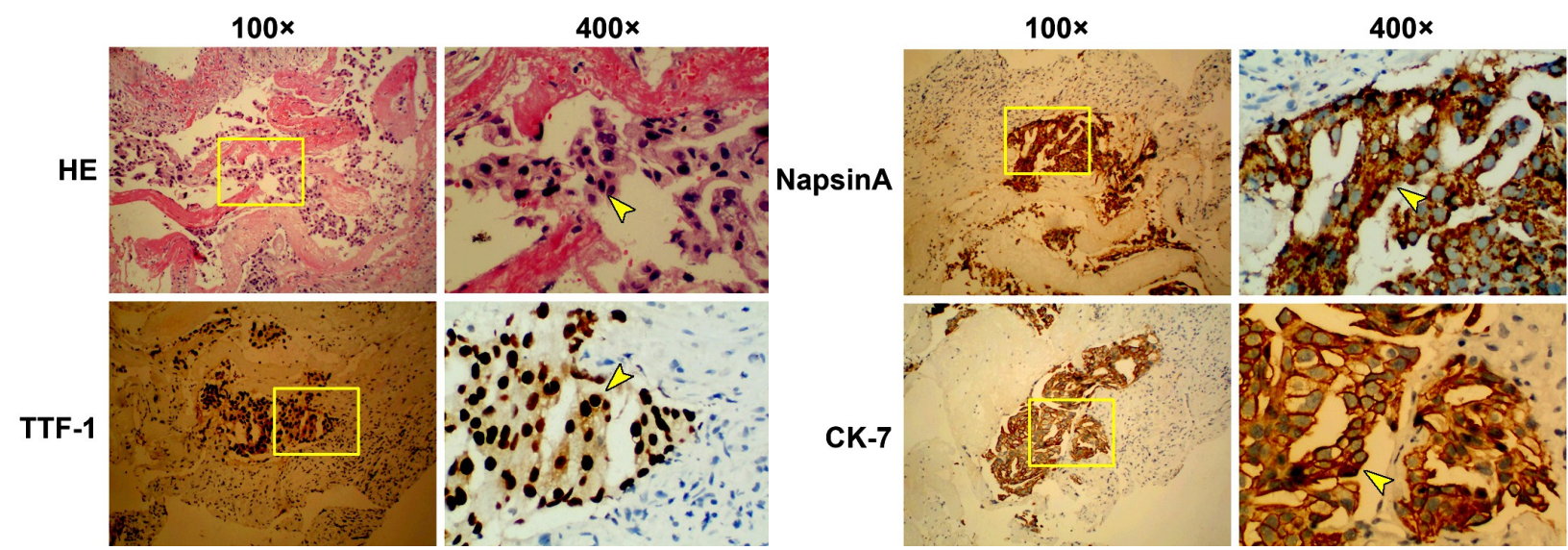

Figure 2. Lung biopsy stained positive for TTF-1, NapsinA, and CK-7. Representative histologic images of lung tissue are shown by H\&E staining, and anti-TTF-1, NapsinA, or CK-7 antibodies with hematoxylin counterstaining. High-magnification views (right column, yellow box area in the left column) present tumor cells (indicated by dark hematoxylin-stained nuclei), TTF-1+ cells (nuclei are stained brown), and NapsinA+ cells and CK-7+ cells (cytoplasm is stained brown), which are indicated by yellow arrows.
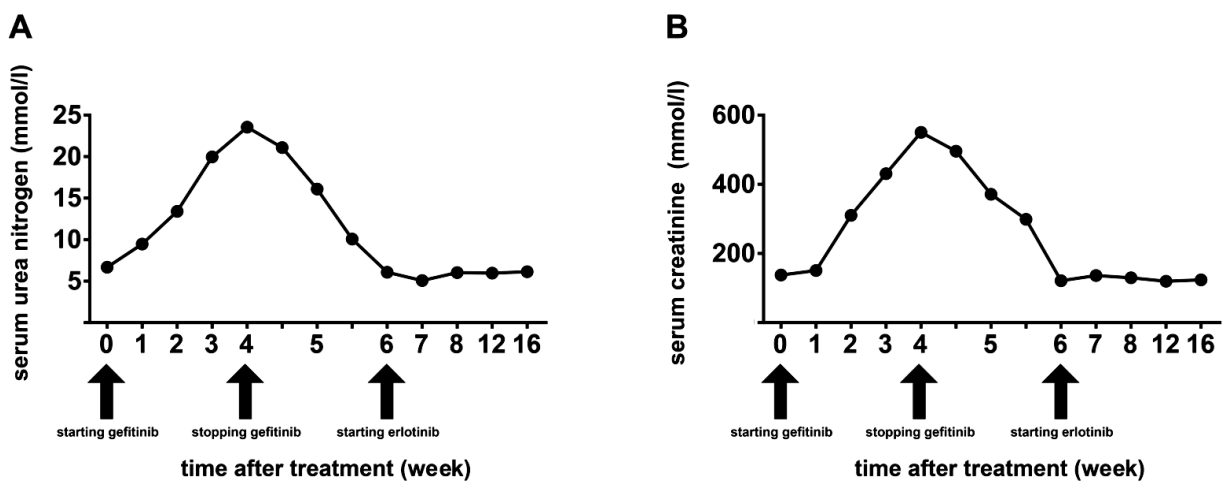

Figure 3. Time course of serum urea nitrogen (A) and creatinine levels (B). Both serum urea nitrogen and creatinine concentrations are increased after starting gefitinib administration, then decreased when treatment was discontinued, while erfitinib treatment had no effect on serum urea nitrogen and creatinine levels.

\section{DISCUSSION}

We have reported successful treatment of a patient with NSCLC and a history of uremia with erlotinib after gefitinib-induced renal dysfunction. The average bioavailability of gefitinib is approximately $60 \%$, the plasma concentration peaks 3-7 $\mathrm{h}$ after oral administration, and the half-life is $48 \mathrm{~h}$. Gefitinib is eliminated primarily by the cytochrome P450 isoenzyme, 3A4 (CYP3A4), in the liver. Eighty-six percent of gefitinib is excreted and $<4 \%$ of gefitinib is excreted by the kidney (11). The most common side effects of a $250 \mathrm{mg}$ daily dose of gefitinib is gastrointestinal and skin toxicities, including diarrhea, nausea, skin rashes, vomiting, asthenia; direct renal toxicity of gefitinib is uncommon (9,11). We treated this patient with $250 \mathrm{mg}$ of gefitinib daily. Because the patient had a history of uremia, we monitored renal function weekly. Within 4 weeks after treatment, the serum urea nitrogen and creatinine levels gradually increased, peaking at $23.6 \mathrm{mmol} / \mathrm{l}$ and $495.6 \mathrm{mmol} / \mathrm{l}$, respectively. Although the patient had a history of uremia, he received hemodialysis and treatment with relevant drugs, and renal function was consistently maintained at a relatively normal level. After gefitinib was discontinued, the serum urea nitrogen and creatinine levels gradually returned to normal levels. Therefore, we consider that the renal dysfunction might have been caused by gefitinib. Similarly, a few cases have been reported 
involving gefitinib-induced renal dysfunction. For example, Maruyama et al. (12) reported that a 57-year-old woman with advanced lung adenocarcinoma developed minimal change nephrotic syndrome after receiving gefitinib, and her symptoms improved completely after discontinuing gefitinib. Another case reported gefitinibassociated membranous nephropathy in a patient with pulmonary adenocarcinoma (13). In addition, Wan et al. (10) reported a case of gefitinib therapy-induced acute renal failure (10).

Erlotinib is another small molecule TKI that targets EGFR and the first-line treatment of patients with locally advanced or metastatic NSCLC with activating mutations of EGFR. A retrospective analysis showed that gefitinib and erlotinib may have nearly identical efficacy in NSCLC patients with activating mutations of EGFR (14). Adverse renal events are extremely rare during erlotinib therapy. Currently, there is only one case report involving erlotinib-induced crescentic glomerulonephritis in a patient with NSCLC [15]. In animal experiments, a number of studies have indicated that erlotinib attenuates the progression of chronic kidney disease, suppresses renal inflammatory cytokine secretion, preserves renal function, and prevents salt retention (16-18). In the current case, after renal function returned to a normal level, erlotinib was initiated and renal function remained stable, which indicated a successful switch to erlotinib therapy after gefitinib-induced renal dysfunction in a patient with EGFR-mutated pulmonary adenocarcinoma and a history of uremia.

In summary, we have reported a patient with an EGFR-mutated pulmonary adenocarcinoma and a history of uremia with gefitinib-induced renal dysfunction and successful treatment with erlotinib.

\section{Ethical approval}

This study was conducted in accordance with the Declaration of Helsinki. This study was conducted with approval from the Ethics Committee of the Affiliated Hospital of Zunyi Medical University. Written informed consent was obtained from the participant.

\section{Declaration of patient consent}

The authors certify that they have obtained all appropriate patient consent forms.

\section{Conflicts of interest: Declared none.}

\section{REFERENCES}

1. Cao M and Chen W (2019) Epidemiology of lung cancer in China. Thorac Cancer, 10: 3-7.

2. Guan WJ, Zheng XY, Chung KF, Zhong NS (2016) Impact of air pollution on the burden of chronic respiratory diseases in China: time for urgent action. Lancet, 388: 1939-51.

3. Molina JR, Yang P, Cassivi SD, Schild SE, Adjei AA (2008) Non-small cell lung cancer: epidemiology, risk factors, treatment, and survivorship. Mayo Clin Proc, 83: 584-94.

4. Rosell R, Moran T, Queralt C, Porta R, Cardenal F, Camps C, et al.; Spanish Lung Cancer Group (2009) Screening for epidermal growth factor receptor mutations in lung cancer. N Engl J Med, 361:958-67.

5. Hsiue EH, Lee JH, Lin CC, Yang JC (2016) Safety of gefitinib in non-small cell lung cancer treatment. Expert Opin Drug Saf, 15: 993-1000.

6. Ciardiello F and Tortora G (2008) EGFR antagonists in cancer treatment. N Engl J Med, 358: 1160-74.

7. Galetti M, Alfieri RR, Cavazzoni A, La Monica S, Bonelli M, Fumarola C, Mozzoni P, De Palma G, Andreoli R, Mutti A, Mor M, Tiseo M, Ardizzoni A, Petronini PG (2010) Functional characterization of gefitinib uptake in non-small cell lung cancer cell lines. Biochem Pharmacol, 80: 179-87.

8. Cataldo VD, Gibbons DL, Pérez-Soler R, Quintás-Cardama A (2011) Treatment of non-small-cell lung cancer with erlotinib or gefitinib. N Engl J Med, 364: 947-55.

9. Cosmai L, Gallieni M, Porta C (2015) Renal toxicity of anticancer agents targeting HER2 and EGFR. J Nephrol, 28: 647 $-57$.

10. Wan HL and Yao NS (2006) Acute renal failure associated with gefitinib therapy. Lung, 184: 249-50.

11. Rawluk J and Waller CF (2018) Gefitinib. Recent Results Cancer Res, 211: 235-46.

12. Maruyama K, Chinda J, Kuroshima T, Kabara M, Nakagawa $\mathrm{N}$, Fujino T, et al. (2015) Minimal change nephrotic syndrome associated with gefitinib and a successful switch to erlotinib. Intern Med, 54: 823-6.

13. Kaneko T, Shimizu A, Aoki M, Tsuruoka S (2015) A case of gefitinib-associated membranous nephropathy in treatment for pulmonary adenocarcinoma. CEN Case Rep, 4: 317.

14. Yoshida T, Yamada K, Azuma K, Kawahara A, Abe H, Hattori $S$, et al. (2013) Comparison of adverse events and efficacy between gefitinib and erlotinib in patients with non-smallcell lung cancer: a retrospective analysis. Med Oncol, 30: 349. 
15. Kurita N, Mise N, Fujii A, Ikeda S, Sugimoto T (2009) Crescentic glomerulonephritis in a patient with advanced lung cancer during erlotinib therapy. NDT Plus, 2: 512-3.

16. Yamamoto $Y$, Iyoda $M$, Tachibana $S$, Matsumoto $K$, Wada Y, Suzuki T, Iseri K1, Saito T1, Fukuda-Hihara K1, Shibata T (2018) Erlotinib attenuates the progression of chronic kidney disease in rats with remnant kidney. Nephrol Dial Transplant, 33: 598-606.
17. Omachi K, Miyakita R, Fukuda R, Kai Y, Suico MA, Yokota $T$, Kamura M, Shuto T, Kai H (2017) Long-term treatment with EGFR inhibitor erlotinib attenuates renal inflammatory cytokines but not nephropathy in Alport syndrome mouse model. Clin Exp Nephrol, 21: 952-60.

18. Bou Matar RN, Klein JD, Sands JM (2013) Erlotinib preserves renal function and prevents salt retention in doxorubicin treated nephrotic rats. PLoS One, 8: e54738. 
